\title{
Effect of asthma exacerbation during pregnancy in women with asthma: a population-based cohort study
}

\author{
Kawsari Abdullah ${ }^{1,2}$, Jingqin Zhu ${ }^{1}$, Andrea Gershon $0^{1,3,4,5,6}$, Sharon Dell ${ }^{1,5,7,8}$ \\ and Teresa To ${ }^{1,3,5}$
}

Affiliations: ${ }^{1}$ Child Health Evaluative Sciences, The Hospital for Sick Children Research Institute, Toronto, ON,

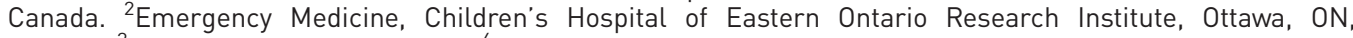
Canada. ${ }^{3}$ ICES, Toronto, ON, Canada. ${ }^{4}$ Division of Respirology, Sunnybrook Health Sciences Centre, Toronto, ON, Canada. ${ }^{5}$ Dalla Lana School of Public Health, University of Toronto, Toronto, ON, Canada. ${ }^{6}$ Dept of Medicine, University of Toronto, Toronto, ON, Canada. ${ }^{7}$ Respiratory Medicine, The Hospital for Sick Children, Toronto, ON, Canada. ${ }^{8}$ Dept of Paediatrics, University of Toronto, Toronto, ON, Canada.

Correspondence: Kawsari Abdullah, Children's Hospital of Eastern Ontario Research Institute, 401 Smyth Road, Ottawa K1H 8L1, Canada. E-mail: KAbdullahlacheo.on.ca

@ERSpublications

This study shows that women with asthma having asthma exacerbation during pregnancy have increased risk of adverse maternal and child health outcomes. Women with asthma during pregnancy require appropriate asthma management and follow-up. http://bit.ly/2Nh8q61

Cite this article as: Abdullah $\mathrm{K}$, Zhu J, Gershon A, et al. Effect of asthma exacerbation during pregnancy in women with asthma: a population-based cohort study. Eur Respir J 2020; 55: 1901335 [https://doi.org/ 10.1183/13993003.01335-2019].

ABSTRACT The association between asthma exacerbation during pregnancy and adverse maternal and child health outcomes have not been investigated appropriately. Our objective was to determine the shortand long-term intergenerational effect of asthma exacerbation in pregnant women with asthma.

A population cohort study was conducted using data from the Ontario asthma surveillance system and population-level health administrative data. Asthma exacerbation in pregnant women with asthma was defined as at least one of the following criteria: at least five physician visits, or one emergency department visit or one hospital admission for asthma during pregnancy. Pregnancy complications, adverse perinatal outcomes and early childhood respiratory disorders were identified using International Classification of Disease codes (9th and 10th revisions).

The cohort consisted of 103424 singleton pregnancies in women with asthma. Asthma exacerbation in pregnant women with asthma was associated with higher odds of pre-eclampsia (OR 1.30, 95\% CI 1.12 1.51 ) and pregnancy-induced hypertension (OR 1.17, 95\% CI 1.02-1.33); babies had higher odds of low birthweight (OR 1.14, 95\% CI 1.00-1.31), preterm birth (OR 1.14, 95\% CI 1.01-1.29) and congenital malformations (OR 1.21, 95\% CI 1.05-1.39). Children born to women with asthma exacerbation during pregnancy had elevated risk of asthma (OR 1.23, 95\% CI 1.13-1.33) and pneumonia (OR 1.12, 95\% CI 1.03-1.22) during the first 5 years of life.

Asthma exacerbation during pregnancy in women with asthma showed increased risk of pregnancy complications, adverse perinatal outcomes and early childhood respiratory disorders in their children, indicating that appropriate asthma management may reduce the risk of adverse health outcomes. 


\section{Background}

Asthma is the most common chronic disease encountered during pregnancy, occurring in $8-13 \%$ of pregnant women worldwide [1]. Nearly $40 \%$ of pregnant women decrease or stop taking asthma medications due to concerns regarding the safety of asthma medications [2,3]. Poorly controlled asthma may increase asthma severity and the risk of having asthma exacerbation during pregnancy [1, 4-7]. Evidence has found that one in three women with asthma suffer from exacerbation during pregnancy $[4,5]$. The key priority in asthma management during pregnancy is maintenance of optimal asthma control.

Various factors may contribute to the development of asthma exacerbation during pregnancy, such as pre-existing severe asthma, non-adherence to controller medication, viral infections, obesity, parity, smoking, environmental smoke and outdoor air pollutants [8-11]. Additionally, risk factors related to health disparities such as specific ethnicity, low socioeconomic and immigrant status may play a role $[9,12]$.

Asthma exacerbation during pregnancy has been found to be associated with adverse perinatal and pregnancy outcomes such as low birthweight, being small for gestational age (SGA), preterm delivery, congenital malformation, pre-eclampsia and perinatal mortality $[1,5,8,11,13]$. However, studies reporting these associations were small cohorts comparing asthmatic women with non-asthmatics during pregnancy. Furthermore, studies using a cross-sectional design have reported that asthma exacerbation during pregnancy can lead to increased risk of asthma, bronchiolitis, atopic dermatitis and allergic rhinitis in their children during early childhood [14-18]. To date, no study has longitudinally followed babies born to women with asthma experiencing asthma exacerbation during pregnancy and investigated the impact of asthma exacerbation on children's respiratory health during early childhood.

Considering the high incidence of asthma exacerbation during pregnancy and the tendency of women to stop taking asthma medication during pregnancy, it is important to examine the possible short- and long-term impacts of this dangerous health condition. Therefore, the objective of this study is to comprehensively evaluate the effect of asthma exacerbations in pregnant women with asthma on maternal and child health in a diverse multiethnic population-based cohort.

\section{Methods}

Data source and ethics consideration

Population-based data from the province of Ontario, Canada, were used for this study. Ontario has a universal, single-payer healthcare system which covers all physician and hospital services for the province's 14 million residents. Five health administrative databases were used in this study: 1) the Ontario Health Insurance Plan (OHIP) database, which contains information on all fee-for-service billings for physician services rendered; 2) the National Ambulatory Care Reporting System database (NACRS), which contains data for hospital-based and community-based emergency and ambulatory care (e.g. day surgery and outpatient clinics); 3) the Ontario Registered Persons Database (RPBD), which includes information on sex, date of birth and residence postal code; 4) the Mother-Baby (MOMBABY) database, which contains information on admission records for mothers and their babies was derived by linking inpatient records of delivering mothers and their newborns, collected by the Canadian Institute for Health Information; and 5) Ontario's Better Outcomes Registry and Network (BORN) database, which collects data on every birth and young child in Ontario. The MOMBABY database was used to identify babies born to mothers with asthma exacerbation during pregnancy. The BORN database was used to identify the perinatal and respiratory health outcome of mothers and their children (www.bornontario.ca/en/index.aspx).

Records in these databases were individually linked using Ontario residents' unique, encrypted health card numbers. This linkage protects individuals' identity while allowing for examination of residents' health service use across multiple health administrative databases. These health administrative databases are housed at ICES in Toronto, Canada. This study was approved by the research ethics board at the Hospital for Sick Children (Toronto).

\section{Study design and population}

This is a longitudinal population-based cohort study where mother-baby pairs were identified from health administrative databases and their health outcomes studied from April 1, 2003 to March 31, 2012 (latest follow-up date: March 31, 2015)

\section{Mother cohort}

The mother cohort included women aged between 13 and 45 years with prevalent asthma during pregnancy who had at least one pregnancy resulting in a live or still birth between fiscal year (FY) 2006 and FY2012. Women with multiple births, without OHIP coverage for a period of 14 months prior to pregnancy, who were missing data on age, residence postal code and non-identifiable in the MOMBABY database were excluded. 
Baby cohort

Babies born to women with prevalent asthma during pregnancy were identified from the MOMBABY database and were followed from birth to 5 years of age. Stillborn babies and those who died before age 5 years were excluded from the cohort. We excluded multiple births, as the intrauterine physiology underlying maternal-fetal transmission differs between multiples and singletons and multiple pregnancies have significant risk complications [13].

\section{Measures}

Asthma case definition

The Ontario Asthma Surveillance Information System (OASIS) is a population-based, longitudinal surveillance system that uses health administrative data to identify and track individuals living with asthma in Ontario [19]. It uses a validated case definition of asthma to monitor its prevalence and incidence in the Ontario population. This case definition of at least two asthma physician visits within two consecutive years or at least one asthma hospitalisation yielded $89 \%$ sensitivity and $72 \%$ specificity in children (aged $0-17$ years) and $84 \%$ sensitivity and $76 \%$ specificity in adults (aged $\geqslant 18$ years) [19-21]. Once entered into the database, patients remained part of the asthma population until they moved out of the province or died in order to be consistent with previous evidence indicating that asthma, once diagnosed, may remit, but does not resolve $[22,23]$.

\section{Exposure: maternal asthma exacerbation}

The primary exposure was occurrence of asthma exacerbation during pregnancy. Currently there is no validated health administrative definition of asthma exacerbation. We used the modified version of a definition used by BLAIS and co-workers $[6,13]$. We defined asthma exacerbation as at least one of the following conditions: general practitioner (GP) visits for asthma at least five times, or one emergency department visit for asthma or one hospital admission for asthma during the pregnancy.

Outcomes were as follows. 1) Maternal pregnancy-related complications: pre-eclampsia, pregnancy-induced hypertension (PIH) and maternal death were identified from the MOMBABY database; and 2) perinatal adverse outcomes: outcomes identified in the babies include low birthweight, SGA, preterm babies, congenital malformation, transient tachypnoea of the newborn (TTN) and respiratory distress syndrome (RDS); and 3) early childhood respiratory disorders: incidence of respiratory health outcomes (allergy, wheeze, asthma, bronchiolitis and pneumonia) during the first 5 years since birth were captured from the Ontario health administrative databases.

All maternal pregnancy-related complications, perinatal adverse and early childhood respiratory outcomes are defined by International Classification of Disease (ICD) 9th and 10th revisions (supplementary tables E1, E2 and E3).

\section{Covariates}

We adjusted our analysis by a number of covariates that may be confounding the association between asthma exacerbation and health outcomes, including mother's age, parity, maternal smoking during pregnancy, rural residence and socioeconomic status. Socioeconomic status was determined using the Ontario Marginalisation Index (ON-Marg; www.ontariohealthprofiles.ca/onmargON.php) [24]. ON-Marg is a census-based, geographically derived index that consists of four domains: residential instability, material deprivation, ethnic concentration and dependency. Each domain is measured by quintiles, where the fifth quintile reflects the greatest magnitude of marginalisation (i.e. the most marginalised) and the first quintile reflects the least magnitude. Other covariates included baby's sex and comorbid conditions. Comorbid conditions were adjusted for using the condensed aggregated diagnostic groups (CADGs) that included cardiovascular disease, chronic obstructive pulmonary disease, diabetes, hypertension, lung cancer and nonlung cancers. CADGs are person-focused, diagnosis-based method of categorising subjects' illnesses that are based on ICD codes [25]. We assessed potential effect modifiers (maternal age and maternal smoking) on the primary association investigated in this study (between perinatal outcomes/ maternal pregnancy complication/respiratory outcomes in children and maternal asthma exacerbation during pregnancy) [17].

\section{Statistical analysis}

Descriptive statistics were performed to present the distribution of exposure variables, outcome variables, and covariates of the study. Chi-squared test for categorical variables and t-test for continuous variables were used to assess differences between women with asthma with and without asthma exacerbation during pregnancy. In addition, we estimated the proportion of all outcomes (perinatal, pregnancy complication and respiratory disorders) in women with asthma who did and did not have asthma exacerbation during pregnancy. 
Association between asthma exacerbation in women with asthma (during pregnancy) and perinatal outcomes and pregnancy complications were investigated using logistic regression analysis with generalised estimating equation (GEE) for repeated measures [26]. The GEE models take into account that a women could contribute more than one pregnancy to the analysis by estimating the correlation between consecutive pregnancies [6]. All odds ratios were presented with $95 \%$ confidence intervals after adjusting for potential confounding variables. Separate multivariable regression models were developed for each of the pregnancy and perinatal outcomes.

Poisson regression analysis with GEE for repeated measures was performed to estimate the risk of early childhood respiratory conditions and asthma exacerbation in women with asthma during pregnancy. The number of observed days for each child was used as the offset variable. Estimates of relative risk (RR) with 95\% confidence intervals are reported. Each respiratory outcome was modelled in a separate Poisson regression analysis.

Effect modification was assessed by including interaction terms between relevant risk factors (maternal age and smoking) and asthma exacerbation [27]. We considered an interaction term to be significant if it had a p-value of $<0.3$. Only significant interaction terms were considered to be included in the models.

All analyses were performed using SAS Enterprise Guide 6.1 (SAS Institute, Cary, NC, USA). Control of confounding and reporting of results was performed using guidance given in LEDERER et al. [28]

\section{Results}

The cohort consisted of 103424 singleton pregnancies in 58524 women with asthma. A total of 4455 pregnancies had asthma exacerbation in 2663 women with asthma.

Table 1 depicts the characteristics of pregnancies with asthma exacerbation in women with asthma ( $\mathrm{n}=103424)$. Significantly greater proportions of women with asthma exacerbation were aged $34-45$ years (17.8\% versus $16.7 \%, \mathrm{p}=0.002)$, smoked during pregnancy $(25.6 \%$ versus $19.1 \%, \mathrm{p} \leqslant 0.001)$ and were in the highest quintile (most deprived) of the material deprivation $(22.3 \%$ versus $18.1 \%, \mathrm{p} \leqslant 0.001)$ and residential instability $(21.4 \%$ versus $17.0 \%, \mathrm{p} \leqslant 0.001)$ dimension of the ON-Marg index.

\section{Maternal-related complications}

Figure 1 shows pregnancy complication in women with asthma who did and did not have asthma exacerbation during pregnancy. A significantly greater proportion of pregnancies with asthma exacerbation had pre-eclampsia (5.32\% versus 3.84\%, $\mathrm{p}<0.001)$ and $\mathrm{PIH}(7 \%$ versus $5.37 \%, \mathrm{p}<0.001)$. Table 2 and figure 2 show the results of the multivariable logistic regression model adjusted for potential confounders. Compared to women with asthma who did not have asthma exacerbation during pregnancy, adjusted odds ratio for pre-eclampsia was 1.30 (95\% CI 1.12-1.51; $\mathrm{p}<0.001$ ) and for PIH 1.17 (95\% CI 1.02-1.33; $\mathrm{p}=0.022$ ) in women with asthma having asthma exacerbation during pregnancy.

\section{Adverse perinatal outcomes}

Figure 1 shows perinatal outcomes in women with asthma who did and did not have asthma exacerbation during pregnancy. Babies born to mothers who experienced asthma exacerbation during pregnancy had higher proportion of adverse perinatal outcomes: low birthweight (6.76\% versus $5.28 \%, \mathrm{p}<0.001)$, SGA ( $2.45 \%$ versus $1.89 \%, \mathrm{p}=0.008)$, preterm baby $(8.15 \%$ versus $6.66 \%, \mathrm{p}<0.001)$ and congenital malformation (6.2\% versus $4.97 \%, \mathrm{p}<0.001)$. After adjusting for confounding and testing of effect modification, table 2 and figure 2 show comparison to women with asthma who did not have asthma exacerbation during pregnancy, the adjusted odds ratio for low birthweight was 1.14 (95\% CI 1.00-1.31; p=0.049), for preterm birth 1.14 (95\% CI 1.01-1.29; $\mathrm{p}=0.036)$ and for congenital malformation 1.21 (95\% CI 1.05-1.39; $\mathrm{p}=0.007$ ) in women with asthma having asthma exacerbation during pregnancy.

\section{Early childhood respiratory disorders}

Slightly higher proportions of children with allergy (16.93\% versus $15.72 \%, \mathrm{p}=0.051)$, asthma $(37.77 \%$ versus $31.39 \%, \mathrm{p} \leqslant 0.001)$, bronchiolitis $(39.72 \%$ versus $39.05 \%, \mathrm{p}=0.382)$ and pneumonia $(24.38 \%$ versus $21.69 \%, p \leqslant 0.001)$ were born to women with asthma having asthma exacerbation during pregnancy. In case of wheeze, there was a higher proportion in children born to women with asthma who did not have asthma exacerbation ( $83.19 \%$ versus $84.72 \%$ ). The adjusted multivariable Poisson regression showed elevated risk ratios for asthma (RR 1.23, 95\% CI 1.13-1.33; p<0.001) and pneumonia (RR 1.12, 95\% CI $1.03-1.22 ; \mathrm{p}<0.006)$ in women with asthma having asthma exacerbation during pregnancy (table 3 ).

For all outcome assessment, no significant interaction between maternal age and smoking was identified with asthma exacerbation. 
TABLE 1 Characteristics of pregnancies with and without asthma exacerbation in women with asthma ( $n=103424)$

\begin{tabular}{|c|c|c|}
\hline & No asthma exacerbation & Asthma exacerbation \\
\hline Cases & 98969 & 4455 \\
\hline \multicolumn{3}{|l|}{ Maternal characteristics } \\
\hline \multicolumn{3}{|l|}{ Age category years } \\
\hline $13-17$ & 3798 (3.84) & $206(4.62)$ \\
\hline $18-34$ & $78678(79.50)$ & $3454(77.53)$ \\
\hline $34-45^{*}$ & $16493(16.66)$ & 795 (17.85) \\
\hline Parity & $1(0-18)$ & $1(0-11)$ \\
\hline Smoking during pregnancy* & $16868(19.19)$ & 973 (25.59) \\
\hline Rurality* & $11553(11.70)$ & $610(13.70)$ \\
\hline \multicolumn{3}{|l|}{ ON-Marg index } \\
\hline \multicolumn{3}{|l|}{ Dependency quintile } \\
\hline 1 & 27783 (28.07) & $1240(27.83)$ \\
\hline 2 & 23039 (23.28) & 1013 (22.74) \\
\hline 3 & $18569(18.76)$ & $857(19.24)$ \\
\hline 4 & $15292(15.45)$ & $661(14.84)$ \\
\hline 5 & $13042(13.18)$ & $629(14.12)$ \\
\hline \multicolumn{3}{|l|}{ Material deprivation quintile } \\
\hline 1 & $25250(25.51)$ & 1004 (22.54) \\
\hline 2 & $19983(20.19)$ & 862 (19.35) \\
\hline 3 & $18213(18.40)$ & 783 (17.58) \\
\hline 4 & $16407(16.58)$ & 756 (16.97) \\
\hline $5^{*}$ & $17872(18.06)$ & 995 (22.33) \\
\hline \multicolumn{3}{|l|}{ Ethnic concentration quintile } \\
\hline 1 & $10760(10.87)$ & $470(10.55)$ \\
\hline 2 & $15245(15.40)$ & $678(15.22)$ \\
\hline 3 & $17856(18.04)$ & 815 (18.29) \\
\hline 4 & $22345(22.58)$ & 995 (22.33) \\
\hline 5 & 31519 (31.85) & $1442(32.37)$ \\
\hline \multicolumn{3}{|l|}{ Residential instability quintile } \\
\hline 1 & 22643 (22.88) & $808(18.14)$ \\
\hline 2 & 21570 (21.79) & $863(19.37)$ \\
\hline 3 & $16556(16.73)$ & $777(17.44)$ \\
\hline $4^{*}$ & $20132(20.34)$ & 999 (22.42) \\
\hline $5^{*}$ & $16824(17.00)$ & 953 (21.39) \\
\hline \multicolumn{3}{|l|}{ Baby characteristics } \\
\hline \multicolumn{3}{|l|}{ Sex } \\
\hline Female & 47893 (48.93) & $2129(48.36)$ \\
\hline Male & $49993(51.07)$ & $2273(51.64)$ \\
\hline Birthweight g & 3405 (276-8639) & $3366.50(329-5605)$ \\
\hline
\end{tabular}

Data are presented as $\mathrm{n}, \mathrm{n}$ (\%) or median (range). ON-MARG index: Ontario Marginalisation Index. *: $p<0.05$.

\section{Discussion}

\section{Adverse perinatal outcomes and pregnancy complications}

From our large cohort study including $>100000$ pregnancies we found that the odds of pre-eclampsia to be $30 \%$ higher and the odds of PIH $17 \%$ higher in women with asthma who experienced asthma exacerbation during pregnancy. In babies born to women with asthma who experienced asthma exacerbation during pregnancy, the odds of having low birthweight and preterm was $14 \%$ higher and the odds of having congenital malformation was $21 \%$ higher. Our study did not identify any association with SGA, TTN and RDS.

Our results are consistent with findings from a previous systematic review where pregnant women with asthma exacerbation were compared to pregnant women without asthma exacerbation for pregnancy complications and adverse perinatal outcomes [1, 2, 8, 29]. However, most of the studies in the review were small cohorts with varying definitions of asthma severity and comparison groups (e.g. some had compared asthmatic women with women without asthma). Our study is the first to use population-level data to measure the association between asthma exacerbation during pregnancy and adverse perinatal outcomes and pregnancy complications. Furthermore, our study has compared women with asthma who 


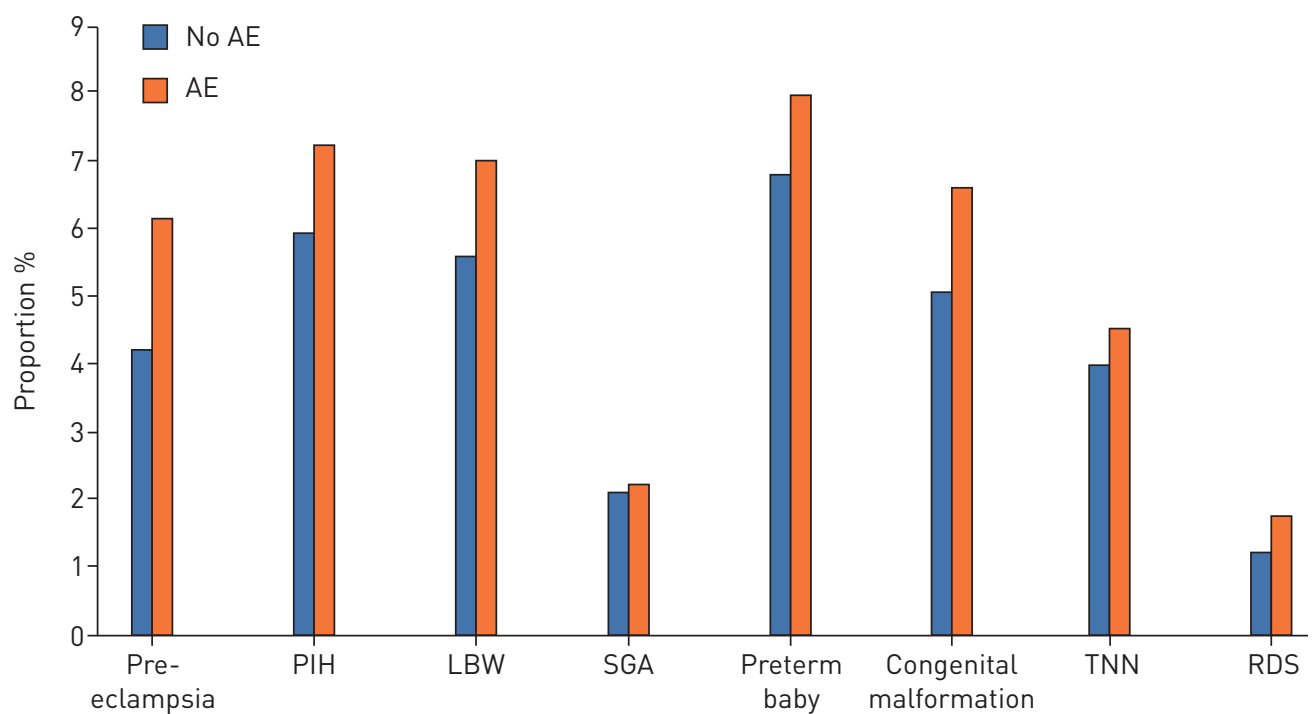

FIGURE 1 Proportion of adverse perinatal outcomes and pregnancy complications in women with asthma who did and did not have asthma exacerbation (AE) during pregnancy. PIH: pregnancy-induced hypertension; LBW: low birthweight; SGA: small for gestational age; TTN: transient tachypnoea of the newborn; RDS: respiratory distress syndrome.

experienced exacerbation during pregnancy to those who have asthma but did not experience exacerbation during pregnancy. Thus, our results denote the impact of asthma exacerbation during pregnancy, as opposed to the impact of asthma.

\section{Effect on respiratory health of children born to mothers experiencing asthma exacerbation during pregnancy}

In the second part of our study, we aimed to identify the long-term impact of asthma exacerbation during pregnancy. We found that children born to women with asthma having asthma exacerbation during pregnancy had $23 \%$ higher risk of developing asthma before the age of 5 years. Previous studies have reported that children whose mothers had asthma exacerbation during pregnancy had increased risk of developing asthma during early childhood $[15,18]$. These studies were performed using population-level data, and similar to our study had compared asthmatic women with asthma exacerbation during pregnancy to those without asthma exacerbation. Hence, our results corroborate with previous evidence. However, our study is the first to show the intergenerational impact of asthma exacerbation during pregnancy by following babies born to women with asthma up to the age of 5 years and evaluating the impact of asthma exacerbation during pregnancy on early childhood respiratory health.

TABLE 2 Risk associated with pregnancy complications and adverse perinatal outcomes in women with asthma who experienced asthma exacerbation during pregnancy

\begin{tabular}{|c|c|c|c|c|c|c|c|c|c|c|}
\hline & \multicolumn{4}{|c|}{ Pregnancy complications ${ }^{\#}$} & \multicolumn{6}{|c|}{ Adverse perinatal outcomes $\rrbracket$} \\
\hline & \multicolumn{2}{|c|}{ Pre-eclampsia } & \multicolumn{2}{|l|}{$\mathrm{PIH}$} & \multicolumn{2}{|c|}{ LBW } & \multicolumn{2}{|c|}{ РTB } & \multicolumn{2}{|c|}{$\begin{array}{l}\text { Congenital } \\
\text { malformation }\end{array}$} \\
\hline $\begin{array}{l}\text { Asthma exacerbation } \\
\text { during pregnancy }\end{array}$ & $\begin{array}{c}1.30 \\
(1.12-1.51)\end{array}$ & $<0.001$ & $\begin{array}{c}1.17 \\
(1.02-1.33)\end{array}$ & 0.022 & $\begin{array}{c}1.14 \\
(1.00-1.31)\end{array}$ & 0.049 & $\begin{array}{c}1.14 \\
(1.01-1.29)\end{array}$ & 0.036 & $\begin{array}{c}1.21 \\
(1.05-1.39)\end{array}$ & 0.007 \\
\hline
\end{tabular}

PIH: pregnancy-induced hypertension; LBW: low birthweight; PTB: preterm birth. \#: adjusted covariates: maternal age, parity, maternal smoking, Ontario Marginalisation Indices (residential instability, material deprivation, ethnic concentration and dependency), collapsed ambulatory diagnostic groups lacute minor, acute major, likely to recur, chronic medical unstable, chronic medical stable, chronic speciality stable, eye/dental, chronic specialty unstable, psychosocial/psychophysiological and prevention/administrative); ${ }^{\uparrow}$ : adjusted covariates: maternal age, parity, maternal smoking, sex of child, Ontario Marginalisation indices (residential instability, material deprivation, ethnic concentration and dependencyl. 


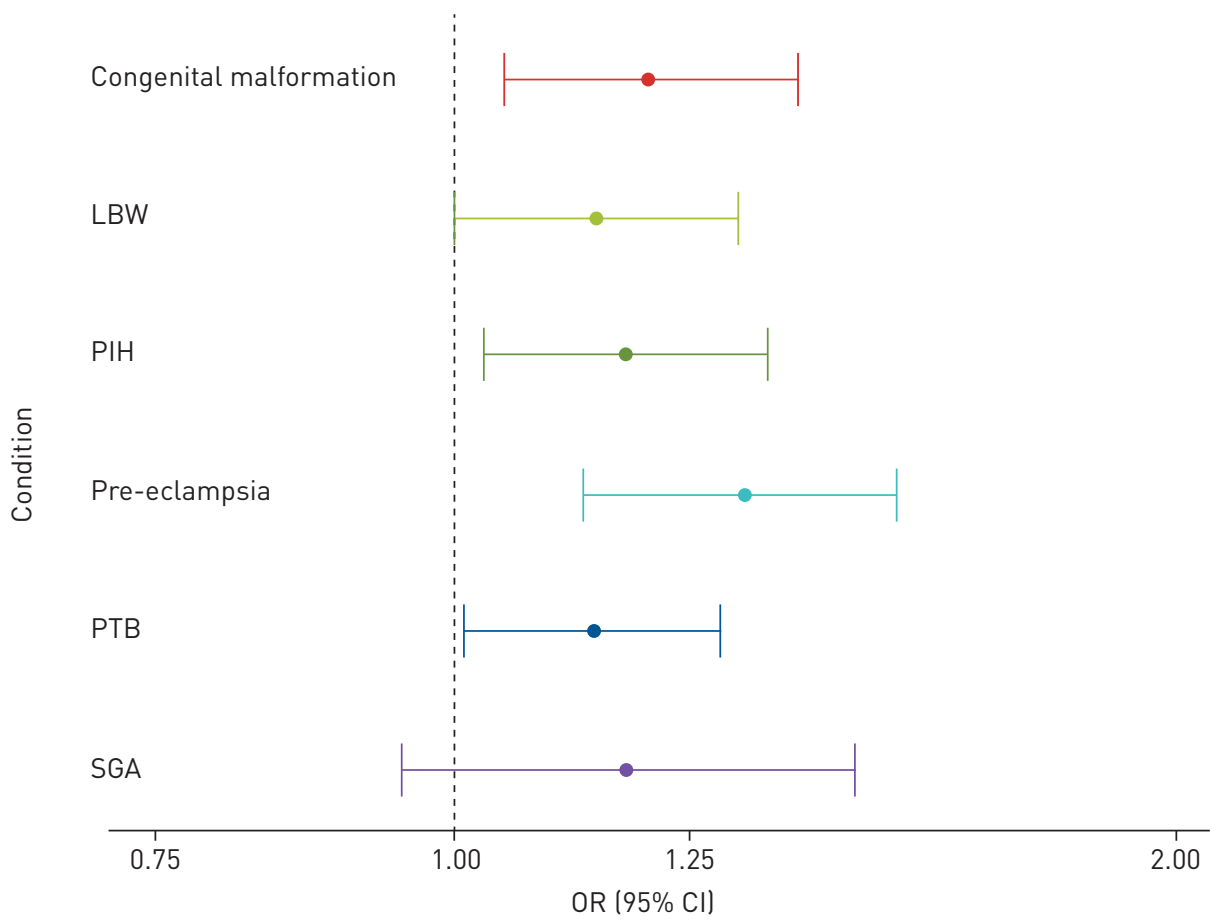

FIGURE 2 Risk of developing pregnancy complications and adverse perinatal outcomes in women with asthma having asthma exacerbation during pregnancy. LBW: low birthweight; PIH: pregnancy-induced hypertension; PTB: preterm birth; SGA: small for gestational age.

In addition, our study identified children born to women with asthma having asthma exacerbation during pregnancy to be at $12 \%$ higher risk of having pneumonia during first 5 years of life. This finding has not been reported in previous studies. However, previous evidence has shown children of mothers with uncontrolled asthma during pregnancy to be more at risk for other respiratory infections such as bronchiolitis and allergic rhinitis [14, 17]. Hence, further research is needed to ascertain this association in the paediatric population.

TABLE 3 Risk associated with developing respiratory disorders in children of women with asthma, experiencing asthma exacerbation during pregnancy

\begin{tabular}{|c|c|c|c|c|c|}
\hline & \multirow[t]{2}{*}{ Cases n } & \multicolumn{2}{|c|}{ Unadjusted } & \multicolumn{2}{|c|}{ Adjusted } \\
\hline & & $\operatorname{RR}(95 \% \mathrm{CI})$ & p-value & RR $(95 \% \mathrm{CI})$ & p-value \\
\hline Allergy $\#$ & 15788 & $1.09(1.00-1.18)$ & 0.0506 & $1.06(0.96-1.16)$ & 0.245 \\
\hline Wheeze & 83902 & $0.98(0.94-1.01)$ & 0.1949 & $1.02(0.98-1.06)$ & 0.344 \\
\hline Asthmaף & 31536 & $1.36(1.26-1.47)$ & $<0.0001$ & $1.23(1.13-1.33)$ & $<0.001$ \\
\hline Bronchiolitis $^{+}$ & 38925 & $1.04(0.98-1.10)$ & 0.2164 & $1.03(0.96-1.10)$ & 0.400 \\
\hline Pneumonia $^{+}$ & 21723 & $1.16(1.08-1.25)$ & $<0.0001$ & $1.12(1.03-1.22)$ & 0.006 \\
\hline
\end{tabular}

RR: relative risk. ${ }^{\#}$ : adjusted for sex of the baby, low birthweight (LBW) baby, small-for-gestational-age baby, preterm baby, Apgar score in $1 \mathrm{~min}$, admitted to intensive care unit (ICU) after birth, history of transient tachypnoea of the newborn (TTN), history of respiratory distress syndrome (RDS), maternal age at delivery, maternal smoking during pregnancy, parity, history of maternal asthma, rurality, dependency quintile, material deprivation quintile, ethnic concentration quintile, residential instability quintile, maternal pre-eclampsia during pregnancy and maternal pregnancy-induced hypertension during pregnancy; ": adjusted for sex of the baby, LBW baby, preterm baby, Apgar score in $1 \mathrm{~min}$, admitted to ICU after birth, history of TTN, history of RDS, parity, history of maternal asthma, rurality, material deprivation quintile, ethnic concentration quintile and residential instability quintile; ${ }^{+}$: adjusted for sex of the baby, LBW baby, preterm baby, admitted to ICU after birth, maternal age at delivery, smoking during pregnancy, parity, history of maternal asthma, rurality, dependency quintile, deprivation quintile, ethnic concentration quintile and residential instability quintile. 
The strengths of this study include the prospective longitudinal design and inclusion of 58524 motherchild pairs and $>100000$ pregnancies. Following babies (born to women with asthma experiencing asthma exacerbation during pregnancy) up to the age of 5 years has allowed us to demonstrate the intergenerational effect of asthma exacerbation during pregnancy. The data linkage across multiple administrative databases ensures that the assessment of outcome is free from individual recall bias. We used validated case definition of asthma as well as ICD codes to ascertain other health outcomes. Our risk estimates were adjusted for a multitude of potential confounders (supplementary tables E4 and E5) including maternal smoking and comorbidities; however, residual confounding from unmeasured confounders may still exist.

There are some limitations of our study. Currently there are no validated algorithms for asthma exacerbation. We defined asthma exacerbation based on a modified version of the algorithm used by BLAIS et al. [13], which includes one filled prescription of oral corticosteroids or one emergency department visit for asthma or one hospital admission for asthma during the length of pregnancy. Validation of our algorithm is beyond the scope of our study.

Using at least five GP visits as a criterion for asthma exacerbation in women with asthma may have the potential to misclassify well-monitored women as having asthma exacerbation. However, previous evidence has shown four or more outpatient visits with an asthma code to be a flag for persistent asthma [30]. Hence by using five or more GP visits as a criterion for asthma exacerbation, we have reduced the potential for misclassification. The higher number of GP visits for asthma is more likely for asthma exacerbation or poorly controlled asthma than diligent monitoring. Future study is needed to validate this case definition to establish its sensitivity and specificity.

We did not categorise asthma exacerbation according to its severity. Based on the work performed by BLAIs et al. [13], we expect our sample to include moderate-to-severe asthma exacerbation groups. Had we adjusted for severity, while our results would not have been grossly different, the magnitude of our findings may have differed.

The use of asthma medications, such as oral corticosteroids during pregnancy may contribute to the development of these adverse outcomes [31-33]. Due to lack of medication data in the Ontario health administrative databases we were unable to incorporate the effect of asthma medication on perinatal and pregnancy outcomes. However, a severe asthma attack probably presents a greater risk to the fetus than the use of asthma medications, because it can provoke maternal hypoxia, which, in addition to decreased placental blood flow, will reduce oxygen supply to the fetus $[1,8,34]$

\section{Conclusion}

Our population-based cohort study identified the detrimental effects of asthma exacerbation during pregnancy. In addition, using a prospective design this study has captured the long-term intergenerational effect of asthma exacerbation during pregnancy by following the same babies up to the age of 5 years. Targeting women with asthma during pregnancy and ensuring appropriate asthma management and postpartum follow-up may help to reduce the risk of pregnancy complications, adverse perinatal outcomes and early childhood respiratory disorders.

Conflict of interest: None declared.

Support statement: This study was supported by ICES, which is funded by an annual grant from the Ontario Ministry of Health and Long-Term Care (MOHLTC). The opinions, results and conclusions reported in this article are those of the authors and are independent from the funding sources. No endorsement by ICES or the Ontario MOHLTC is intended or should be inferred. Parts of this material are based on data and information compiled and provided by CIHI. However, the analyses, conclusions, opinions and statements expressed herein are those of the author, and not necessarily those of CIHI. Funding information for this article has been deposited with the Crossref Funder Registry.

\section{References}

1 Namazy JA, Murphy VE, Powell H, et al. Effects of asthma severity, exacerbations and oral corticosteroids on perinatal outcomes. Eur Respir J 2013; 41: 1082-1090.

2 Murphy VE, Namazy JA, Powell H, et al. A meta-analysis of adverse perinatal outcomes in women with asthma. BJOG 2011; 118: 1314-1323.

3 Enriquez R, Wu P, Griffin MR, et al. Cessation of asthma medication in early pregnancy. Am J Obstet Gynecol 2006; 195: 149-153.

4 Schatz M, Dombrowski MP, Wise R, et al. Asthma morbidity during pregnancy can be predicted by severity classification. J Allergy Clin Immunol 2003; 112: 283-288.

5 Murphy VE, Gibson P, Talbot PI, et al. Severe asthma exacerbations during pregnancy. Obstet Gynecol 2005; 106 : 1046-1054.

6 Blais L, Forget A. Asthma exacerbations during the first trimester of pregnancy and the risk of congenital malformations among asthmatic women. J Allergy Clin Immunol 2008; 121: 1379-1384. 
7 Boulet LP, Becker A, Bérubé D, et al. Canadian Asthma Consensus Report, 1999. Canadian Asthma Consensus Group. CMAJ 1999; 161: Suppl. 11, S1-S61.

8 Murphy VE, Clifton VL, Gibson PG. Asthma exacerbations during pregnancy: incidence and association with adverse pregnancy outcomes. Thorax 2006; 61: 169-176.

9 Ali Z, Ulrik CS. Incidence and risk factors for exacerbations of asthma during pregnancy. J Asthma Allergy 2013; 6: 53-60.

10 Murphy VE, Clifton VL, Gibson PG. The effect of cigarette smoking on asthma control during exacerbations in pregnant women. Thorax 2010; 65: 739-744.

11 Schatz M, Zeiger RS, Hoffman CP, et al. Perinatal outcomes in the pregnancies of asthmatic women: a prospective controlled analysis. Am J Respir Crit Care Med 1995; 151: 1170-1174.

12 Forno E, Celedón JC. Health disparities in asthma. Am J Respir Crit Care Med 2012; 185: 1033-1035.

13 Blais L, Kettani FZ, Forget A, et al. Asthma exacerbations during the first trimester of pregnancy and congenital malformations: revisiting the association in a large representative cohort. Thorax 2015; 70: 647-652.

14 Martel M-J, Beauchesne M-F, Malo J-L, et al. Maternal asthma, its control and severity in pregnancy, and the incidence of atopic dermatitis and allergic rhinitis in the offspring. J Pediatr 2009; 155: 707-713.

15 Martel MJ, Rey É, Beauchesne MF, et al. Control and severity of asthma during pregnancy are associated with asthma incidence in offspring: two-stage case-control study. Eur Respir J 2009; 34: 579-587.

16 Martel MJ, Rey É, Malo JL, et al. Determinants of the incidence of childhood asthma: a two-stage case-control study. Am J Epidemiol 2009; 169: 195-205.

17 Carroll KN, Gebretsadik T, Griffin MR, et al. Maternal asthma and maternal smoking are associated with increased risk of bronchiolitis during infancy. Pediatrics 2007; 119: 1104-1112.

18 Tegethoff M, Olsen J, Schaffner E, et al. Asthma during pregnancy and clinical outcomes in offspring: a national cohort study. Pediatrics 2013; 132: 483-491.

19 Gershon AS, Wang C, Guan J, et al. Identifying patients with physician-diagnosed asthma in health administrative databases. Can Respir J 2009; 16: 183-188.

20 To T, Dell S, Dick PT, et al. Case verification of children with asthma in Ontario. Pediatr Allergy Immunol 2006; 17: 69-76.

21 To T, Wang C, Guan J, et al. What is the lifetime risk of physician-diagnosed asthma in Ontario, Canada? Am J Respir Crit Care Med 2010; 181: 337-343.

22 Stern DA, Morgan WJ, Halonen M, et al. Wheezing and bronchial hyper-responsiveness in early childhood as predictors of newly diagnosed asthma in early adulthood: a longitudinal birth-cohort study. Lancet 2008; 372: 1058-1064

23 van Den Toorn LM, Prins JB, Overbeek SE, et al. Adolescents in clinical remission of atopic asthma have elevated exhaled nitric oxide levels and bronchial hyperresponsiveness. Am J Respir Crit Care Med 2000; 162: 953-957.

24 Matheson F, Dunn J, Smith K, et al. Ontario Marginalization Index User Guide. Version 1.0. Toronto, Centre for Research on Inner City Health, 2012.

25 Weiner JP, Starfield BH, Steinwachs DM, et al. Development and application of a population-oriented measure of ambulatory care case-mix. Med Care 1991; 29: 452-472.

26 Zeger SL, Liang KY, Albert PS. Models for longitudinal data: a generalized estimating equation approach. Biometrics 1988; 44: 1049-1060.

27 Bennett JA. Mediator and moderator variables in nursing research: conceptual and statistical differences. Res Nurs Health 2000; 23: 415-420.

28 Lederer DJ, Bell SC, Branson RD, et al. Control of confounding and reporting of results in causal inference studies. Guidance for authors from editors of respiratory, sleep, and critical care journals. Ann Am Thorac Soc 2019; 16: 22-50.

29 Murphy VE, Wang G, Namazy JA, et al. The risk of congenital malformations, perinatal mortality and neonatal hospitalisation among pregnant women with asthma: a systematic review and meta-analysis. BJOG 2013; 120: 812-822.

30 Schatz M, Zeiger RS, Yang S-JT, et al. Persistent asthma defined using HEDIS versus survey criteria. Am J Manag Care 2010; 16: e281-e288.

31 Bracken MB, Triche EW, Belanger K, et al. Asthma symptoms, severity, and drug therapy: a prospective study of effects on 2205 pregnancies. Obstet Gynecol 2003; 102: 739-752.

32 Dombrowski MP, Schatz M, Wise R, et al. Asthma during pregnancy. Obstet Gynecol 2004; 103: 5-12

33 Schatz M, Dombrowski MP, Wise R, et al. The relationship of asthma medication use to perinatal outcomes. J Allergy Clin Immunol 2004; 113: 1040-1045.

34 Clifton VL, Giles WB, Smith R, et al. Alterations of placental vascular function in asthmatic pregnancies. Am J Respir Crit Care Med 2001; 164: 546-553. 